\title{
Estilos de Apego en Relaciones de Pareja y su Asociación con la Satisfacción Marital
}

\author{
Attachment Styles in Couples and Their \\ Association With Marital Satisfaction
}

\author{
Mónica Guzmán y Paula Contreras \\ Universidad Católica del Norte
}

\begin{abstract}
El objetivo del estudio fue evaluar diferencias en la satisfacción marital en función de los estilos de apego y el efecto de interacción entre el estilo de apego propio y el de la pareja sobre la satisfacción en 129 participantes y sus respectivas parejas, provenientes de la ciudad de Antofagasta, Chile. Se realizó un estudio transversal, con diseño no experimental y muestra de conveniencia, basado en mediciones de auto-reporte del Experiences in Close Relationships y el Marital Satisfaction Scale. Mediante ANOVA factorial, los resultados indican que las personas con estilo de apego seguro son las que reportan los niveles de satisfacción más altos y las con estilos desentendidos, los más bajos. En las díadas la combinación de ambos miembros de la pareja con estilos seguros es la que está asociada a los niveles de satisfacción más altos y la combinación desentendido-temeroso, a los más bajos. No hubo diferencias en la satisfacción según sexo, edad, escolaridad, afiliación religiosa, presencia de hijos ni tiempo de la relación marital.
\end{abstract}

Palabras clave: apego, pareja, satisfacción marital

\begin{abstract}
The aim of the study was to evaluate differences in marital satisfaction according to attachment styles and the interaction between the individual and the partner's attachment styles in a sample of 129 participants and their partners, all of them residing in the city of Antofagasta, Chile. A crosssectional study with a non-experimental design with a convenience sample was performed, based on self-report measures of the Experiences in Close Relationships and the Marital Satisfaction Scale. Through factorial ANOVA, the results indicate that secure attachment was related to the highest level of marital satisfaction, while dismissing styles were associated with the lowest levels. In dyads the combination of both partners with secure attachment styles was associated with the highest levels of satisfaction, while the combination of dismissing and fearful attachment was associated with the lowest degree of satisfaction. No differences were found according to gender, age, educational level, religious affiliation, presence of children, or length of the marital relationship.
\end{abstract}

Keywords: attachment, couple, marital satisfaction

La satisfacción en las relaciones de pareja se ha transformado en un tema de preocupación para terapeutas de parejas, investigadores y profesionales del área de la salud, dado los hallazgos que indican que las parejas que mantienen conflictos sostenidos presentan graves consecuencias en su salud física y emocional (Gottman \& Levenson, 1999).

\section{Apego Adulto}

La teoría del apego, cuya formulación fue iniciada por John Bowlby (1969, 1979, 1980), da cuenta de la necesidad humana universal de formar vínculos afectivos estrechos hacia los cuales recurrir en momentos de sufrimiento o estrés. Este constructo es definido como un sistema motivacional que busca mantener la proximidad entre bebés y sus cuidadores como forma de

Mónica Guzmán González y Paula Contreras Garay, Escuela de Psicología, Universidad Católica del Norte, Antofagasta, Chile.

La correspondencia relativa a este artículo debe ser dirigida a Mónica Guzmán González, Escuela de Psicología, Universidad Católica del Norte, Avda. Angamos 0610, Antofagasta, Chile. E-mail: moguzman@ucn.cl 
obtener protección y sobrevivir. Si bien inicialmente la teoría del apego se focalizó de manera prioritaria en las relaciones tempranas, surgió con posterioridad una serie de estudios que aplicaron los principios del apego a la edad adulta. Fueron pioneras en este campo las investigaciones iniciadas por Hazan y Shaver (1987) aplicadas al amor de pareja, quienes sostuvieron que el comportamiento del adulto en relaciones cercanas está moldeado por representaciones mentales, cuyos orígenes se encuentran en las relaciones del niño con sus cuidadores primarios.

Si bien se sostiene que las necesidades de apego son universales, las conductas de apego presentan claras diferencias individuales que pueden ser explicadas en términos de los modelos operativos internos (MOI), formados sobre la base de experiencias repetidas con las figuras significativas (Feeney, 2002). Los MOI pueden ser definidos como representaciones o esquemas que un individuo tiene de sí mismo y los otros (Marrone, 2001), que guían la manera en que se funciona en diversos contextos interpersonales, especialmente aquellos que propician la intimidad.

La imagen de sí mismo está relacionada con el grado en el cual se experimenta ansiedad acerca de ser rechazado o abandonado, de modo tal que las personas que poseen una visión positiva de sí mismas tenderían a experimentar baja ansiedad respecto de esta posibilidad, dado que se consideran dignas de ser amadas y cuidadas. Por el contrario, aquellas personas que poseen una visión negativa de sí mismas tenderían a manifestar preocupación y temor frente al abandono de quien es la figura de apego.

Por su parte, la imagen del otro estaría asociada al grado de evitación que la persona manifiesta respecto de las relaciones cercanas, de manera que aquellos que tienen una imagen positiva del otro en términos de su confiabilidad y disponibilidad, tendrán mayor facilidad para establecer relaciones cercanas con otro. Por el contrario, aquellos que tienen una visión negativa de los demás, como poco receptivos, tenderán a evitar involucrarse más íntimamente en los vínculos. Estas dos dimensiones, ansiedad y evitación, pueden ser medidas con cuestionarios de auto-reporte confiables y válidos y han sido asociadas a distintos aspectos del funcionamiento interpersonal (Mikulincer, Shaver \& Slav, 2006).

Los MOI antes descritos son explícitamente relacionales, es decir, incluyen imágenes de sí y de los otros en situaciones altamente interdependientes, más que en aislamiento (Shaver, Collins \& Clark, 1996). Además, pueden ser usados para predecir el comportamiento en diversos ámbitos del funcionamiento humano, pues sirven como filtros a través de los cuales se interpretan las relaciones con los otros. Ambas dimensiones se articulan en patrones sistemáticos de expectativas, necesidades, emociones, estrategias de regulación emocional y conducta social, denominados estilos de apego (Feeney \& Noller, 2001), que influyen en la manera en la que un individuo se involucra en relaciones cercanas.

Siguiendo los planteamientos de Bowlby respecto de la representación de sí mismo y de los otros, Bartholomew y Horowitz (1991) desarrollaron un modelo de cuatro categorías de apego, a las cuales subyacen dos dimensiones, la ansiedad del abandono y la evitación de la cercanía emocional: (a) seguro, que aúna una idea positiva de sí mismo y de los demás, evidenciando, por tanto, baja ansiedad y evitación ante los contactos interpersonales de mayor intimidad; (b) desentendido o evitativo, con una idea positiva de sí mismo y negativa de los demás y, por lo tanto, manifiesta baja ansiedad y alta evitación; (c) preocupado, con una idea negativa de sí y positiva de los demás, acompañada de alta ansiedad y baja evitación; y (d) temeroso, con una idea negativa tanto de sí como de los otros, asociada a alta ansiedad y alta evitación.

Si bien hay acuerdo en que estos patrones de apego son relativamente estables, dada la tendencia a asimilar la nueva experiencia a los MOI existentes y a involucrarse en estilos interaccionales auto-perpetuantes, también han demostrado ser dinámicos en relación con el contexto y el surgimiento de nuevas relaciones (Bowlby, 1980; Cassidy, 2000).

Dado que los estilos de apego se expresan a lo largo de la vida en nuevas relaciones, es comprensible que jueguen un rol importante en las relaciones de pareja, por ser vínculos que 
favorecen la intimidad y el cuidado mutuo (Fraley \& Shaver, 2000). Hazan y Shaver (1987), quienes desarrollaron las primeras investigaciones en este ámbito, propusieron que las relaciones de pareja pueden ser conceptualizadas como relaciones de apego, las que se encontrarían influidas, en parte, por las experiencias con los cuidadores, internalizadas en los MOI. De acuerdo a estos autores, existiría un paralelo entre el apego del infante y su cuidador y el apego en las relaciones de pareja, expresado en el deseo de mantener la proximidad física, la confianza en la figura de apego para el confort y la visión de esta como una fuente de seguridad en momentos de estrés. Sin embargo, el apego adulto difiere del apego infantil en diversos aspectos: en las relaciones de pareja la naturaleza de la relación es recíproca, las necesidades afectivas previamente satisfechas por los cuidadores se transfieren gradualmente hacia las parejas significativas en la adultez (Fraley \& Shaver, 2000) y el apego adulto se manifiesta en la calidad de las relaciones y en la regulación del afecto ante situaciones de amenaza o conflicto (Feeney \& Noller, 2001; Hazan \& Shaver, 1987).

\section{Apego y Satisfacción en las Relaciones de Pareja}

Desde los primeros trabajos conducidos por Hazan y Shaver (1987), un cuerpo importante de investigaciones ha examinado el vínculo existente entre la seguridad en el apego y la calidad de las relaciones de pareja, siendo el grado de satisfacción con la relación una de las dimensiones más estudiadas, definida en este estudio como la actitud favorable o desfavorable hacia la relación en un momento dado del tiempo (Roach, Frazier \& Bowden, 1981). La evidencia que apoya estos planteamientos es vasta: gran cantidad de estudios han mostrado la asociación entre el apego adulto y la satisfacción, tanto en matrimonios como en parejas solteras (e.g., Collins \& Feeney, 2000; Collins \& Read, 1990; Edwards, 2007; Feeney, 2002; Kirkpatrick \& Davis, 1994; Kobak \& Hazan, 1991; Meyers \& Landsberger, 2002).

En el trabajo de Hazan y Shaver (1987) los autores reportaron que las personas con mayor seguridad en el apego describían sus experiencias amorosas como más felices, amistosas y de confianza. Solían experimentar el amor como un estado que puede tener altibajos, pero que en general se mantiene constante. Además, tendían a tener relaciones de más largo plazo. En contraste, las personas con mayor ansiedad en el apego eran más propensas a experimentar celos, obsesión o atracción sexual extrema. Por su parte, las personas con alta evitación describían sus relaciones caracterizadas por el temor a la cercanía y por frecuentes altibajos emocionales. Por este motivo, las personas inseguras en el apego disfrutan menos de sus relaciones. Por su parte, Feeney y Noller (2001) encontraron que las personas evitativas en el apego eran más propensas a decir que nunca se han enamorado, a no comprometerse y a tener bajos ideales en el amor. En cambio, las personas con alta ansiedad tendieron a reportar más preocupación obsesiva y dependencia emocional del otro. Al contrario, las personas con mayor seguridad en el apego reportaron los mayores niveles de satisfacción, confianza y los menores niveles de expectativas insatisfechas respecto de sus parejas. Collins y Feeney (2000) encontraron que personas con apego inseguro tienden a desplegar menos conductas de cuidado hacia el otro y a tener expectativas más negativas respecto del apoyo de la pareja, lo que redundaría en una menor satisfacción. A similares resultados han llegado estudios más recientes. Por ejemplo, Cohen y Eagle (2005) encontraron que personas con alta ansiedad o evitación del apego reportaron menor consenso, cohesión, expresión emocional y ajuste global en sus relaciones. En un estudio con matrimonios, Butzer y Campbell (2008), quienes investigaron la asociación entre el apego y la satisfacción tanto sexual como con la relación, encontraron que las personas con altos niveles de ansiedad y evitación fueron las que reportaron los menores niveles de satisfacción sexual. Además, se encontró que la relación entre la satisfacción sexual y la marital fue mayor en las personas con alta ansiedad del apego y aquellas que tenían parejas ansiosas en el apego. En las personas con alta evitación las satisfacciones sexual y marital no estuvieron asociadas. 
En Chile se han encontrado resultados en la misma línea. Concretamente, en un estudio conducido con una muestra de matrimonios se encontró que las dimensiones de ansiedad y evitación del apego operan disminuyendo la satisfacción marital (Rivera, 2006).

Resultados levemente divergentes fueron reportados por Hollist y Miller (2005), en el sentido que encontraron que estilos de apego seguros no influían en la cualidad marital, pero que estilos de apego inseguro sí impactaban negativamente en la satisfacción. Es decir, este estudio mostró que la inseguridad del apego era un predictor más poderoso de la satisfacción que la seguridad del apego. Del igual modo, Möller, Hwang y Wickberg (2006) no encontraron en su estudio asociación entre el apego seguro y la satisfacción marital, pero sí entre el apego inseguro y la insatisfacción marital. Por otra parte, al comparar el aporte de las dimensiones de ansiedad y evitación del apego, LeRoy (2008) reportó que la dimensión de evitación estaba más consistentemente asociada con la satisfacción marital que la dimensión de ansiedad, tanto en hombres como en mujeres.

También se ha constatado que la satisfacción marital varía según el estilo de apego de los miembros de la díada, siendo mayor en las díadas seguras que en las inseguras (Banse, 2004; Feeney \& Noller, 2001; Hazan \& Shaver, 1994; Rivera, 2006), y del sexo. Así, por ejemplo, Kirkpatrick y Davis (1994) concluyeron que los hombres que se encontraban con una pareja ansiosa reportaron menor satisfacción que los que estaban con una pareja segura. Simpson (1990), por su parte, encontró que el apego evitativo estaba asociado inversamente con la satisfacción, tanto en hombres como en mujeres, y que el apego ansioso estaba asociado inversamente con la satisfacción, pero solo en mujeres.

Complementando estos hallazgos, Feeney (2002) encontró que la relación entre la seguridad del apego y la satisfacción marital estaba moderada por la conducta percibida en el cónyuge. Específicamente, las personas con estilos de apego inseguro eran más reactivas a las conductas negativas de sus parejas y experimentaban menor satisfacción que las personas seguras en el apego. Este efecto fue más marcado en personas con apegos temerosos (i.e., alta ansiedad y alta evitación) y en aquellas que tenían matrimonios de mayor duración.

En definitiva, los estudios revisados permiten concluir que existe asociación entre las características del apego y la satisfacción marital, siendo más marcada entre los estilos inseguros y la baja satisfacción marital. Una parte de la investigación ha demostrado, además, que dicha asociación está moderada por las características de la pareja. Sin embargo, se requieren más estudios que profundicen en dicha asociación. Por esto, los objetivos principales del presente estudio fueron evaluar las diferencias en la satisfacción marital en función de los estilos de apego y evaluar la interacción entre el estilo de apego propio y el de la pareja en su efecto sobre la satisfacción marital.

\section{Hipótesis}

Las hipótesis formuladas en el estudio fueron:

1. El grado de satisfacción marital varía en función del estilo de apego de las personas. Específicamente, la satisfacción es mayor en personas con estilo de apego seguro que en las con estilo de apego inseguro.

2. Existe un efecto de interacción del estilo de apego propio y el de la pareja en la satisfacción. Específicamente, el efecto negativo del estilo de apego inseguro sobre la satisfacción con la relación se magnifica cuando la pareja tiene a su vez un estilo de apego inseguro. 


\section{Método}

\section{Diseño de Investigación}

Se realizó un estudio de tipo transversal, con un diseño descriptivo-comparativo no experimental, basado en mediciones de auto-reporte, en el que se consideró como variable dependiente el grado de satisfacción experimentado en la relación y como variables independientes el estilo de apego propio y el de la pareja. Dado que en estudios previos se han reportado diferencias en la satisfacción por sexo, presencia de hijos y número de años de la relación (e.g., Feeney, 2002; Kirkpatrick \& Davis, 1994; Simpson, 1990; Vera, Félix-Castro \& Rodríguez-Barrera, 2001), se decidió evaluar preliminarmente estas asociaciones para eventualmente integrar estas variables como un factor adicional en los análisis.

\section{Participantes}

Se evaluó una muestra no probabilística de conveniencia, no clínica, compuesta por 129 participantes y sus respectivas parejas, provenientes de la ciudad de Antofagasta, Chile.

Los criterios de inclusión fueron tener más de 20 años y encontrarse en una relación heterosexual de matrimonio o convivencia de al menos un año de duración. Se estableció este tiempo como mínimo por ser el que se considera necesario para que surjan temáticas vinculadas al apego y para que se desarrolle un cierto sentido de interdependencia y de identidad como pareja (Fincham, Jackson \& Beach, 2005).

Del total de la muestra, 65 (50,4\%) fueron hombres y $64(49,6 \%)$, mujeres, con edades fluctuantes entre los 20 y 60 años y un promedio de edad de 38,9 años $(D E=9,6)$. El promedio de edad de las mujeres fue 37,4 años $(D E=8,6)$ y 40,5 años $(D E=10,3)$ el de los hombres.

Un $62,1 \%$ de los participantes reportó ser casado, un $26,6 \%$, soltero, un $7,3 \%$, separado y un $3,9 \%$, divorciado. El tiempo promedio de relación fue 13,1 años $(D E=9,6)$, con un rango entre 1 y 37 años. Un $28,2 \%$ de los participantes pertenecía a una familia ensamblada.

La gran mayoría de los participantes reportó tener hijos (89,8\%), con un promedio de 2,1 hijos $(D E=0,9)$ y un rango entre 1 y 5 hijos. Respecto de la distribución por edad de los hijos mayores, un 13,2\% tiene hijos entre los 0 y 5 años, un 22,8\% entre los 6 y 12 años, un 24,6\% entre los 13 y 17 años, un 20,2\% entre los 18 y 24 años, un 8,8\% entre los 25 y 30 años y un $10,5 \%$ entre los 31 y 40 años.

Respecto de la afiliación religiosa, un 60,9\% de los participantes se identificó como católico, un 18,8\% señaló no tener ninguna religión, un 14,8\% mencionó ser evangélico, mientras que el porcentaje restante indicó tener otra religión $(3,1 \%)$, ser ateo o agnóstico $(1,6 \%)$ o mormón $(0,8 \%)$.

\section{Instrumentos}

Se aplicó un cuestionario de auto-reporte en el que se incluyeron los siguientes instrumentos:

Experiences in Close Relationships (ECR); Brennan, Clark \& Shaver, 1998). Por medio de este cuestionario de auto-reporte se evaluaron los estilos de apego. Incluye 36 ítems en formato Likert ( 1 = no me representa para nada, $7=$ me representa completamente), que arroja puntajes en dos escalas de 18 ítems cada una: la ansiedad asociada al apego, es decir, el grado 
en que la persona se siente segura o insegura respecto de la disponibilidad de la propia pareja, y la evitación asociada al apego, que evalúa el grado en que la persona se siente cómoda y confortable siendo cercana o dependiendo de otros. La escala de ansiedad incluye ítems tales como "Tengo miedo de ser abandonado/a por una pareja" y "Necesito que mi pareja me confirme constantemente que me quiere". Por su parte, la escala de evitación incluye ítems como "Me siento incómodo/a cuando una pareja trata de acercarse mucho a mí" y "Me cuesta depender emocionalmente de una pareja”. El puntaje en cada escala se obtiene promediando el puntaje de los 18 ítems obtenidos en cada dimensión; un mayor puntaje indica mayor ansiedad y/o evitación.

Para este estudio el cuestionario fue aplicado con la instrucción de responderlo en base a la relación de pareja actual, no en las relaciones románticas en general, como señala la versión original. Tal decisión se fundamentó en lo expresado por Kobak (1999, citado en Martínez \& Santelices, 2005), en que si bien el estilo de apego sería relativamente estable, su manifestación específica sería producto de la interacción entre los propios MOI y la calidad de una relación en particular.

Además de la obtención de un puntaje asociado a cada dimensión, el ECR permite ubicar a las personas en una de cuatro categorías de estilos de apego: un estilo seguro, asociado a bajos niveles de ansiedad y/o evitación, y tres estilos inseguros: preocupado (alta ansiedad, baja evitación), desentendido (baja ansiedad, alta evitación) y temeroso (alta ansiedad y alta evitación).

Dado que al momento de conducir este estudio no estaba disponible la versión validada del instrumento en Chile, se ocupó una versión adaptada por Guzmán (2010).

Los índices de consistencia interna, reportados por Brennan et al. (1998) mediante alfa de Cronbach, son 0,91 y 0,94 para las escalas de ansiedad y evitación, respectivamente. Para la muestra del estudio el alfa de Cronbach fue 0,84 para la escala de ansiedad y 0,83 para la de evitación.

La evidencia de validez de constructo del ECR está basada en estudios que muestran que es un predictor significativo de constructos teóricamente relevantes, tales como la satisfacción marital (Brennan et al., 1998) y la presencia de problemas interpersonales (Wei, Vogel, Ku \& Zakalik, 2005).

Marital Satisfaction Scale (MSS), Roach et al. (1981). Para la evaluación de la satisfacción con la relación se ocupó este cuestionario de auto-reporte que evalúa la actitud favorable o desfavorable hacia la relación en un momento dado del tiempo. Está compuesto por 48 ítems respondidos en formato Likert ( $1=$ muy en desacuerdo, $5=$ muy de acuerdo), con puntajes que fluctúan entre 48 y 240 puntos. Un mayor puntaje indica mayor satisfacción. Ejemplos de ítems son: "Siempre puedo confiar en mi pareja", "Me llevo bien con mi pareja".

Para este estudio se ocupó la versión validada en Chile por Tapia (2005). El punto de corte para la población chilena es de 158 puntos. Esto significa que las personas que están bajo este puntaje pertenecen a la población disfuncional en términos de ajuste marital y las personas que están sobre este puntaje pertenecen a la población funcional en su funcionamiento de pareja.

Este instrumento ha mostrado tener buenas propiedades psicométricas: presentó validez concurrente de 0,79 con la escala de Locke y Wallace (1959, citado en Roach et al., 1981) y obtuvo un índice de consistencia interna, evaluada por alfa de Cronbach, de 0,91 (Roach et al., 1998). Para la muestra del estudio el alfa de Cronbach de la escala fue 0,94. 
Características demográficas. Entre otras variables, en el cuestionario se consignó la edad, sexo, estado civil, número y edad de los hijos, tiempo de relación, actividad y afiliación religiosa.

\section{Procedimiento}

Luego de la firma de un consentimiento informado y de explicar que el objetivo del estudio era conocer de qué manera las personas viven sus relaciones de pareja, cada participante y su respectiva pareja respondieron de manera individual el cuestionario de auto-reporte. El cuestionario fue aplicado por un equipo de ayudantes debidamente capacitados en la aplicación del mismo, quienes se hicieron cargo de proporcionar las instrucciones para que cada pareja lo respondiese en su domicilio y luego lo devolviera.

A todos los participantes se les enfatizó el carácter anónimo y confidencial de sus respuestas.

\section{Análisis de Datos}

Antes de iniciar el análisis de la información se realizó un examen de los datos a fin de detectar casos con valores ausentes. De los 136 casos que inicialmente conformaban la muestra, se detectaron cuatro casos con un número elevado de datos ausentes en alguna de las escalas (> 8\%), por lo que se decidió eliminarlos, tras chequear que fuesen datos omitidos al azar (Hair, Anderson, Tatham \& Black, 1999). Los ítems no respondidos por un participante en alguna de las escalas $(<8 \%)$ fueron reemplazados con la media obtenida en los ítems respondidos por ese participante en la escala correspondiente.

Para detectar casos atípicos multivariados se ocupó la distancia de Mahalanobis como estadístico. Con este análisis, se identificaron tres casos que excedían el valor crítico $(p<0,001)$, por lo cual fueron eliminados de los análisis (Hair et al., 1999). De esta manera, la muestra definitiva del estudio quedó conformada por 129 casos.

Posteriormente se realizaron los análisis descriptivos y se evaluaron las diferencias en la satisfacción según variables socio-demográficas, mediante sucesivos análisis de varianza (ANOVA) y pruebas $t$ de Student.

A fin de examinar si existían diferencias en la satisfacción marital según los diferentes estilos de apego, así como su posible interacción con el estilo de apego de la pareja, se procedió a realizar un análisis de varianza factorial. Se consideró como variable dependiente el grado de satisfacción marital y como variables independientes el estilo de apego propio y el de la pareja.

Los contrastes post hoc se llevaron a cabo con la prueba Games-Howell para varianzas no homogéneas, dado que, al evaluar el supuesto de homogeneidad de las varianzas de la satisfacción marital con el estadístico de Levene, este mostró que las varianzas de los grupos no eran iguales, $F(15,113)=1,86, p=0,04)$.

Si bien 258 personas completaron los instrumentos, es decir, 129 parejas, para los análisis se consideró solo a uno de los miembros de la díada (seleccionado aleatoriamente), agregando como variable adicional a cada persona el estilo de apego de la pareja. Tal decisión se fundamentó en que analizar la muestra total de 258 personas hubiese implicado la violación del supuesto de la independencia de las observaciones requerido para el ANOVA.

Para los análisis de datos, se utilizó el programa SPSS versión 15.0. 


\section{Resultados}

\section{Análisis Descriptivos}

En la Tabla 1 se presenta la estadística descriptiva de las variables evaluadas para la muestra total, así como para hombres y mujeres por separado.

De acuerdo a los promedios observados, se destaca que la muestra en general reportó puntajes relativamente bajos en el componente de ansiedad del apego y promedios más bajos aún en el componente de evitación. Los niveles de satisfacción marital se ubicaron sobre el punto de corte establecido para la población chilena (158 puntos), indicando que la muestra evaluada se encuentra dentro de un nivel funcional. La prueba Kolmogorov-Smirnov indicó que la muestra se distribuye normalmente $(1,18, p=0,13)$. Respecto de hombres y mujeres, el nivel de satisfacción fue levemente más alto en los hombres; sin embargo, la diferencia de los promedios no fue estadísticamente significativa, $t(127)=0,70, p=0,49$.

Tabla 1

Estadística Descriptiva de las Variables Apego y Satisfacción Marital

\begin{tabular}{lcccc}
\hline \multicolumn{1}{c}{ Variable } & Rango & Total & $\begin{array}{c}\text { Mujeres } \\
M(D E)\end{array}$ & $\begin{array}{c}\text { Hombres } \\
M(D E)\end{array}$ \\
\hline Ansiedad asociada al apego & $1-7$ & $3,24(1,10)$ & $3,37(1,13)$ & $3,12(1,07)$ \\
Evitación asociada al apego & $1-7$ & $2,47(0,95)$ & $2,38(0,95)$ & $2,56(0,94)$ \\
Satisfacción marital & $48-240$ & $181,76(28,64)$ & $179,98(26,50)$ & $183,51(30,79)$ \\
\hline
\end{tabular}

$N=129$

En la Tabla 2 se presenta la distribución de frecuencias de los estilos de apego de los participantes y de sus parejas. Como puede observarse, el estilo de apego seguro fue el más frecuente, seguido del estilo preocupado.

Tabla 2

Distribución de Frecuencias de los Estilos de Apego de los Participantes y sus Parejas

\begin{tabular}{lcccc}
\hline \multirow{2}{*}{ Tipo de apego } & \multicolumn{2}{c}{ Participantes } & \multicolumn{2}{c}{ Pareja } \\
\cline { 2 - 5 } & Frecuencia & $\%$ & Frecuencia & $\%$ \\
\hline Seguro & 69 & 53,5 & 57 & 44,2 \\
Preocupado & 33 & 25,6 & 33 & 25,6 \\
Temeroso & 14 & 10,9 & 25 & 19,4 \\
Desentendido & 13 & 10,1 & 14 & 10,9 \\
Total & 129 & 100,1 & 129 & 100,1 \\
\hline
\end{tabular}


Respecto de las combinaciones de apego en las parejas evaluadas, las combinaciones más frecuentes fueron las díadas en que ambos tienen estilo de apego seguro $(n=50)$, seguidas de aquellas en que ambos poseen estilo de apego inseguro $(n=47) \mathrm{y}$, finalmente, aquellas en que uno de los miembros tiene apego seguro y el otro inseguro $(n=32)$.

$\mathrm{Al}$ evaluar diferencias en la satisfacción marital de acuerdo a variables socio-demográficas, los resultados indicaron que no existían diferencias estadísticamente significativas en la satisfacción marital según presencia de hijos, $t(127)=-0,37, p=0,71$, escolaridad, $F(6,120)=1,08$, $p=0,14$, ni afiliación religiosa, $F(5,122)=0,39, p=0,86$. Tampoco hubo correlación entre la satisfacción marital y el número de años de la relación, $r(129)=0,10, p=0,25$, el número de hijos, $r(129)=-0,13, p=0,15$, y la edad, $r(129)=0,11, p=0,21$.

\section{Estilos de Apego y Satisfacción Marital}

Los resultados del ANOVA factorial indican que existen diferencias estadísticamente significativas en la satisfacción marital en función del propio estilo de apego, $F(3,125)=14,12, p$ $<0,001, \eta^{2}=0,27$, pero no en función del estilo de apego de la pareja, $F(3,125)=2,41, p=0,07$. Los contrastes post hoc con la prueba Games Howell indican que tales diferencias se dan entre las personas con estilos de apego seguro, $M=192,34(D \mathrm{E}=7,02), 95 \%$ IC [184,45, 200,23], y aquellas con estilos inseguros: temeroso, $M=157,71(D \mathrm{E}=3,98), 95 \%$ IC $[143,81,171,61]$, preocupado, $M=172,93(D \mathrm{E}=4,90), 95 \%$ IC $[163,23,182,64]$ y desentendido, $M=145,09(D \mathrm{E}$ $=6,14), 95 \%$ IC $[132,92,157,26]$, reportando mayor satisfacción con la relación quienes son seguros en el apego $(p<0,001)$. Dentro del grupo de los estilos inseguros, las personas con patrones de apego preocupado expresan mayor satisfacción con la relación que aquellas con apego desentendido, siendo estas últimas las que reportan los niveles más bajos (Ver Figura 1). Estos resultados apoyan la primera hipótesis del estudio.

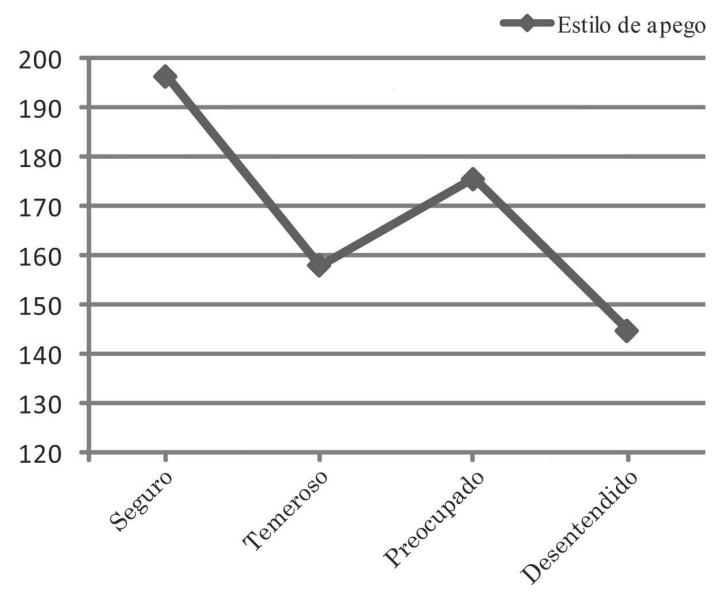

Figura 1. Estilos de apego del participante y satisfacción marital. $N=129$.

Por otra parte, al analizar el efecto de interacción entre el estilo de apego propio y el de la pareja, se observa que este es estadísticamente significativo, $F(15,113)=2,16, p=0,03$. Ello indica que el efecto del estilo de apego propio sobre la satisfacción marital variaría dependiendo del estilo de apego de la pareja. El tamaño del efecto observado fue de $\eta^{2}=0,13$, con una potencia estadística de 0,83 (Cohen, 1992). 
Al realizar las comparaciones de los efectos simples en las configuraciones diádicas (ver Figura 2), en el caso de quienes poseen un estilo de apego seguro, sus niveles de satisfacción se mantuvieron relativamente estables y elevados con independencia del estilo de apego de la pareja $(p=0,11)$. Sin embargo, cuando la persona poseía un estilo de apego inseguro, el efecto de esta variable sobre la satisfacción se vio amplificado o minimizado, dependiendo del estilo de apego de la pareja. Así, cuando se posee un estilo de apego desentendido, el estar con una pareja con estilo temeroso tuvo el efecto más negativo sobre la satisfacción $(p=0,02), M=124$ $(D E=9,63), 95 \%$ IC $[104,93,143,07]$, magnificándose este, al igual que la combinación de una persona con apego temeroso con una pareja con estilo desentendido $(p=0,03), M=129,8(D E=$ 10,76), 95\% IC [108,43, 151,07]. Estos resultados apoyan la segunda hipótesis del estudio.

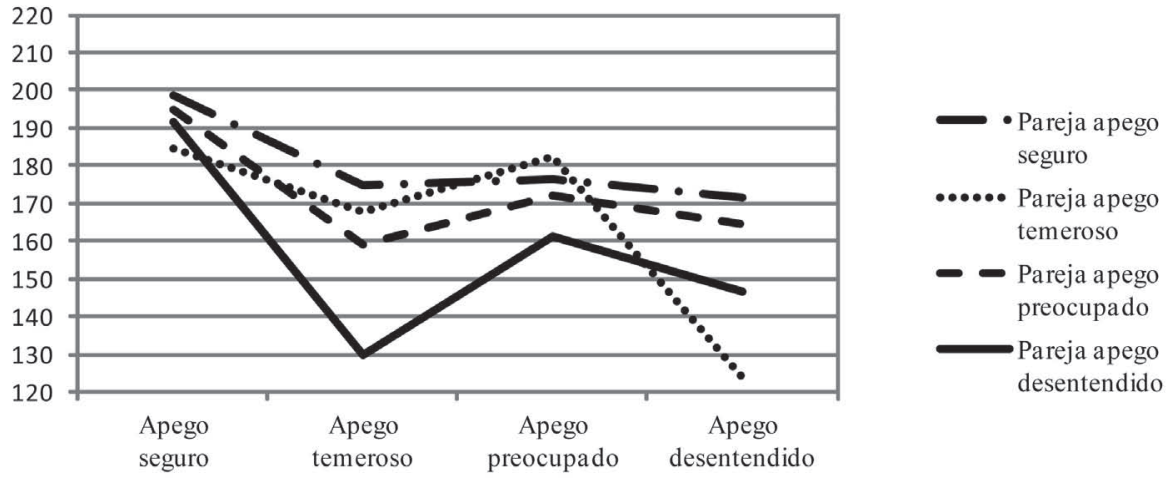

Figura 2. Interacción entre los estilos de apego del participante y su pareja y su asociación con la satisfacción marital. En el eje horizontal del gráfico se presenta el estilo de apego propio.

\section{Discusión}

Los resultados del presente estudio sugieren algunas reflexiones interesantes. Los hallazgos muestran que ser hombre o mujer no tiene relación con el grado de satisfacción al interior de la relación. Desde el punto de vista psicosocial y clínico, este aspecto cobra sentido a la luz de estudios en terapia de pareja que señalan que son las mujeres las que más se quejan de la relación de pareja respecto de los hombres. Según Tapia (2001), en la mayoría de las discusiones de pareja es la mujer la que se queja o critica al hombre y el hombre es el que se defiende o calla. Respecto de este punto, se podría pensar, entonces, que las diferencias entre hombres y mujeres determinan perspectivas diferentes, diversas visiones de mundo, pero no necesariamente mayor o menor satisfacción al interior de la relación de pareja. Tampoco se observaron diferencias significativas entre las personas que tienen o no tienen hijos. Al respecto, si bien Vera et al. (2001) concluyen que los matrimonios perciben un decremento en su satisfacción marital conforme aumenta el número de hijos y los años de relación, estos resultados difieren de lo encontrado en este estudio. Considerando la gran sobrecarga de roles y multifuncionalidad que viven las parejas de hoy, especialmente las mujeres, se podría pensar que las parejas que no tienen hijos tendrían menor probabilidad de presentar estrés, por tanto, mayor satisfacción en la relación. Sin embargo, este hallazgo hace suponer que el punto no tiene relación con tener o no hijos, sino en cómo la pareja vive la co-parentalidad. La falta de asociación encontrada en este estudio entre los años de relación y la satisfacción podría apoyar la noción de que la calidad de una relación no está asociada necesariamente al tiempo que una pareja lleva junta. Sin embargo, para llegar a conclusiones más claras se hace necesario desarrollar estudios con diseños longitudinales que permitan evaluar más precisamente estos aspectos. 
En relación a las diferencias en la satisfacción marital según el estilo de apego propio, el resultado referido a los niveles más altos de satisfacción obtenidos por los estilos seguros y los más bajos por los desentendidos son coherentes con hallazgos de investigaciones previas que indican que personas seguras en el apego tienden a experimentar mayor bienestar y satisfacción en sus relaciones (e.g., Collins \& Feeney, 2000; Collins \& Read, 1990; Edwards, 2007; Feeney, 2002; Kirkpatrick \& Davis, 1994; Kobak \& Hazan, 1991).

Tal resultado podría interpretarse a la luz de los modelos positivos de sí y de los otros que subyacen a los estilos seguros y que podrían traducirse en que la pareja sea vivenciada como más respetuosa, responsiva y cuidadosa y a que se esté más atento/a a las claves positivas de la relación, (e.g., Collins \& Feeney, 2004). En la misma línea, Kirkpatrick y Hazan (1994) han reportado que las personas con estilos de apego seguro tienen relaciones con mejor comunicación, proporcionan mayor apoyo a sus parejas y resuelven de manera más constructiva sus conflictos. Adicionalmente, los estudios indican que las personas con estilos de apego seguro controlan sus sentimientos negativos de un modo relativamente constructivo, reconociendo su ansiedad y buscando apoyo o consuelo en la pareja (Pietromonaco, Greenwood \& Barrett, 2004).

En cambio, en el caso de los estilos inseguros, los resultados son coherentes con la literatura previa que indica que tanto la ansiedad como la evitación del apego están asociadas a la percepción de la pareja como menos capaz de brindar apoyo y a evaluaciones más negativas respecto del involucramiento, intimidad, compromiso y amor del otro (e.g., Butzer \& Campbell, 2008; Collins \& Feeney, 2004).

Por otra parte, la obtención de los niveles más bajos de satisfacción de los apegos desentendidos podría comprenderse a partir de las estrategias de desactivación de las necesidades de apego, propias de las personas con estos estilos. Así, la negación de las necesidades emocionales y la evitación de la intimidad generarían un terreno menos propicio para obtener gratificación en sus relaciones de pareja. Asimismo, su tendencia a la evasión en situaciones de necesidad (en lugar de buscar apoyo) no dan la oportunidad a que la pareja se constituya en una fuente efectiva de contención emocional, lo cual dificultaría que se sintieran más satisfechas en sus relaciones. Además, se ha visto que a las personas con alta evitación les es difícil enamorarse (Hazan \& Shaver, 1987).

Respecto del comportamiento de las díadas, los mayores niveles de satisfacción obtenidos por la combinación de ambos miembros de la díada con estilo seguro indicaría el efecto benéfico y potenciador de la relación cuando ambos experimentan comodidad con la cercanía y dependencia y bajo temor al abandono y/o rechazo. Tal inclinación permitiría disfrutar más de los aspectos positivos de la relación. Sin embargo, los efectos negativos de un estilo inseguro sobre la satisfacción se hacen aún más intensos si la pareja tiene a su vez un estilo inseguro. Dicho efecto alcanza el nivel más alto cuando se tiene un estilo de apego desentendido y se está con una pareja con estilo de apego temeroso, lo que podría entenderse a partir de la tendencia a tomar distancia emocional propia de los estilos desentendidos, con dificultad para comprometerse. En ese contexto el estar con una pareja que es ambivalente respecto del compromiso e involucramiento emocional, con una excesiva demanda a momentos pero, al mismo tiempo, con temor a mostrarse en sus aspectos más íntimos y vulnerables podría hacer que la relación sea vivenciada como menos gratificante.

En definitiva, los resultados de este estudio apoyan la relevancia y pertinencia de incorporar una perspectiva relacional en la comprensión de la satisfacción marital, pues esta no solo tendría que ver con las características propias, sino también con las de la pareja y de lo que emerge en ese espacio vincular.

Desde una perspectiva clínica, estos resultados invitan a pensar en un abordaje terapéutico que, incorporando la teoría del apego, permita responder a la demanda de ayuda de las parejas en conflicto que buscan un espacio en el que puedan comprender sus necesidades afectivas en 
un contexto protegido. En ese sentido, un aporte significativo es la propuesta de la Terapia de Pareja Centrada en las Emociones de Susan Johnson (2008) y la Terapia Narrativa de Apego de Arlene Vetere y Rudi Dallos (2008), cuyo eje central articulador es la teoría del apego adulto y la perspectiva sistémica.

La Terapia de Pareja Centrada en las Emociones se ha desarrollado como un modelo de terapia de parejas basada en la evidencia y el estudio de sus resultados terapéuticos ha validado sistemáticamente su eficacia y capacidad para producir cambios duraderos (Baucom, Shoham, Mueser, Daiuto \& Stickle, 1998; Butler, Harper \& Mitchell, 2011). Este modelo de terapia se focaliza en la experiencia emocional del drama interpersonal de una pareja, promoviendo el desarrollo de un apego más firme, que nutra y fortalezca a ambos miembros de la relación. Desde esta perspectiva, es fundamental contar con sistemas e instrumentos de medición del apego que permitan diseñar intervenciones focalizadas para cada pareja. En ese sentido, el presente estudio aporta antecedentes a favor de lo expuesto anteriormente: la necesidad de desarrollar intervenciones diferenciales.

De este modo, la creación de nuevas interacciones que redefinan la relación como una fuente de seguridad y apoyo emocional para cada uno de los miembros de la pareja rompe, entonces, con las posiciones rígidas de las categorías y clasificaciones, para poner en el espacio interaccional el cómo los estilos de apego de la díada se expresan en una relación en particular, favoreciendo u obstaculizando el bienestar y la satisfacción con el otro.

Por su parte, la Terapia Narrativa de Apego integra la teoría del apego, la teoría de los sistemas familiares y las teorías narrativas en un enfoque integrado para la práctica clínica de la terapia sistémica (Vetere \& Dallos, 2008). Esta terapia enfatiza la construcción de un contexto terapéutico que incorpore la creación de una base segura, la exploración de narrativas y la promoción de experiencias de apego dentro de un marco sistémico. Un aporte interesante de esta terapia es la inclusión del terapeuta en la construcción de una base segura al interior del sistema terapéutico. Así, la creación de esta base estable en la construcción del vínculo terapéutico se define como la primera oportunidad para procesar y reprocesar la experiencia emocional, a través de la expresión de intenciones, deseos y miedos en un ambiente seguro.

Si se toma en cuenta lo propuesto por estos dos modelos de intervención que tienen en su centro la teoría del apego adulto, ambos suponen desafíos importantes a la hora de desarrollar el trabajo clínico con parejas: el desarrollo de intervenciones diferenciales, que vayan más allá de lo estipulado en manuales de tratamiento, y el rol de terapeuta para fomentar el desarrollo de una base segura. En ese sentido, la investigación sobre la relación entre las características del apego romántico y diversos aspectos del funcionamiento en relaciones de pareja cobra especial relevancia.

Finalmente, si bien este estudio aporta antecedentes para una comprensión más relacional del efecto de los estilos de apego sobre la satisfacción marital, posee algunas limitaciones que deben ser consideradas. En primer lugar, los resultados de esta investigación no son generalizables a otras muestras, por ejemplo, parejas más jóvenes o en relaciones de noviazgo. Segundo, es probable que haya un sesgo en la muestra a favor de personas para quienes las temáticas de pareja eran más salientes o se encontraban experimentando menos conflictos en su relación. Por último, la evaluación mediante cuestionarios de auto-reporte requiere de quienes responden cierta capacidad de introspección. En consideración de lo anterior, estudios futuros podrían evaluar si resultados similares se mantienen en otro tipo de parejas, con otros métodos de evaluación, así como examinar cambios en el comportamiento de las díadas en el tiempo, mediante diseños longitudinales. 


\section{Referencias}

Banse, R. (2004). Adult attachment and marital satisfaction: Evidence for dyadic configuration effects. Journal of Social and Personal Relationships, 21, 273-282. doi:10.1177/0265407504041388

Bartholomew, K. \& Horowitz, L. M. (1991). Attachment styles among young adults: A test of a four-category model. Journal of Personality and Social Psychology, 61, 226-244. doi:10.1037/0022-3514.61.2.226

Baucom, D. H., Shoham, V., Mueser, K. T., Daiuto, A. D. \& Stickle, T. R. (1998). Empirically supported couple and family interventions for marital distress and adult mental health problems. Journal of Consulting and Clinical Psychology, 66, 53-88. doi:10.1037/0022-006X.66.1.53

Bowlby, J. (1969). El vínculo afectivo. Buenos Aires, Argentina: Paidós.

Bowlby, J. (1979). Vínculos afectivos: formación, desarrollo y pérdida. Madrid, España: Morata.

Bowlby, J. (1980). La pérdida afectiva. Buenos Aires, Argentina: Paidós.

Brennan, K. A., Clark, C. L. \& Shaver, P. R. (1998). Self-report measurement of adult attachment: An integrative overview. En J. A. Simpson \& W. S. Rholes (Eds.), Attachment theory and close relationships (pp. 46-76). New York, NY: Guilford Press.

Butler, M. H., Harper, J. M. \& Mitchell, C. B. (2011). A comparison of attachment outcomes in enactment-based versus therapist-centered therapy process modalities in couple therapy. Family Process, 50, 203-220. doi:10.1111/j.15455300.2011.01355.x

Butzer, B. \& Campbell, L. (2008). Adult attachment, sexual satisfaction, and relationship satisfaction: A study of married couples. Personal Relationships, 15, 141-154. doi:10.1111/j.1475-6811.2007.00189.x

Cassidy, J. (2000). Adult romantic attachments: A developmental perspective on individual differences. Review of General Psychology, 4, 111-131. doi:10.1037/1089-2680.4.2.111

Cohen, J. (1992). A power primer. Psychological Bulletin, 112, 155-159. doi:10.1037/0033-2909.112.1.155

Cohen, A. \& Eagle, M. (2005). Prediction of relational functioning from attachment in adult romantic relationships. Journal of the American Psychoanalytic Association, 53, 1331-1333.

Collins, N. L. \& Feeney, B. C. (2000). A safe haven: An attachment theory perspective on support seeking and caregiving in intimate relationships. Journal of Personality and Social Psychology, 78, 1053-1073. doi:10.1037/00223514.78.6.1053

Collins, N. L. \& Feeney, B. C. (2004). Working models of attachment shape perceptions of social support: Evidence from experimental and observational studies. Journal of Personality and Social Psychology, 87, 363-383. doi:10.1037/0022-3514.87.3.363

Collins, N. L. \& Read, S. J. (1990). Adult attachment, working models, and relationship quality in dating couples. Journal of Personality and Social Psychology, 58, 644-663. doi:10.1037/0022-3514.58.4.644

Edwards, J. K. (2007). Relationship satisfaction: The role of attachment, conflict, empathy and forgiveness (Tesis de Doctorado no publicada), Purdue University, West Lafayette, IN, Estados Unidos.

Feeney, J. A. (2002). Attachment, marital interaction, and relationship satisfaction: A diary study. Personal Relationships, 9, 39-55. doi:10.1111/1475-6811.00003

Feeney, J. \& Noller, P. (2001). Apego adulto. Bilbao, España: Desclée De Brouwer.

Fincham, F. D., Jackson, H. \& Beach, S. R. H. (2005). Transgression severity and forgiveness: Different moderators for objective and subjective severity. Journal of Social and Clinical Psychology, 24, 860-875. doi:10.1521/ jscp.2005.24.6.860

Fraley, R. C. \& Shaver, P. R. (2000). Adult romantic attachment: Theoretical developments, emerging controversies, and unanswered questions. Review of General Psychology, 4, 132-154. doi:10.1037/1089-2680.4.2.132

Gottman, J. M. \& Levenson, R. W. (1999). Rebound from marital conflict and divorce prediction. Family Process, 38, 287-292. doi:10.1111/j.1545-5300.1999.00287.x

Guzmán, M. (2010). Apego y perdón de transgresiones relacionales ocurridas en la pareja: el rol mediador de las atribuciones negativas y la satisfacción (Tesis de Doctorado no publicada), Pontificia Universidad Católica de Chile, Santiago, Chile.

Hair, J. F., Anderson, R. E., Tatham, R. L. \& Black, W. C. (1999). Análisis multivariante (5a ed.). Madrid, España: Pearson Educación.

Hazan, C. \& Shaver, P. (1987). Romantic love conceptualized as an attachment process. Journal of Personality and Social Psychology, 52, 511-524. doi:10.1037/0022-3514.52.3.511

Hazan, C. \& Shaver, P. R. (1994). Attachment as an organizational framework for research on close relationships. Psychological Inquiry, 5, 1-22. doi:10.1207/s15327965pli0501_1

Hollist, C. S. \& Miller, R. B. (2005). Perceptions of attachment style and marital quality in midlife marriage. Family Relations, 54, 46-57. doi:10.1111/j.0197-6664.2005.00005.x

Johnson, S. (2008). Práctica de la Terapia Matrimonial Concentrada Emocionalmente. New York, NY: Routledge.

Kirkpatrick, L. A. \& Davis, K. E. (1994). Attachment style, gender, and relationship stability: A longitudinal analysis. Journal of Personality and Social Psychology, 66, 502-512. doi:10.1037/0022-3514.66.3.502

Kirkpatrick, L. A. \& Hazan, C. (1994). Attachment styles and close relationships: A four-year prospective study. Personal Relationships, 1, 123-142. doi:10.1111/j.1475-6811.1994.tb00058.x

Kobak, R. R. \& Hazan, C. (1991). Attachment in marriage: Effects of security and accuracy of working models. Journal of Personality and Social Psychology, 60, 861-869. doi:10.1037/0022-3514.60.6.861

LeRoy, D. (2008). Factors that influence the association between adult attachment and marital satisfaction (Tesis de Doctorado no publicada), Utah State University, Logan, UT, Estados Unidos. 
Marrone, M. (2001). La teoría del apego. Madrid, España: Editorial Psimática.

Martínez, C. \& Santelices, M. P. (2005). Evaluación del apego en el adulto: una revisión. Psykhe, 14(1), $181-191$. doi:10.4067/S0718-22282005000100014

Meyers, S. A. \& Landsberger, S. A. (2002). Direct and indirect pathways between adult attachment style and marital satisfaction. Personal Relationships, 9, 159-172. doi:10.1111/1475-6811.00010

Mikulincer, M., Shaver, P. R. \& Slav, K. (2006). Attachment, mental representations of others, and gratitude and forgiveness in romantic relationships. En M. Mikulincer \& G. S. Goodman (Eds.), Dynamics of romantic love: Attachment, caregiving, and sex (pp. 190-215). New York, NY: Guilford Press.

Möller, K., Hwang, C. P. \& Wickberg, B. (2006). Romantic attachment, parenthood and marital satisfaction. Journal of Reproductive and Infant Psychology, 24, 233-240. doi:10.1080/02646830600821272

Pietromonaco, P. R., Greenwood, D. \& Barrett, L. F. (2004). Conflict in adult close relationships: An attachment perspective. En W. S. Rholes \& J. A. Simpson (Eds.), Adult attachment: Theory, research, and clinical implications (pp. 267-299). New York, NY: Guilford Press.

Rivera, D. (2006). Influencia de los estilos de apego y habilidades pro relacionales en la satisfacción y bienestar emocional en relaciones de pareja (Tesis de Doctorado no publicada), Pontificia Universidad Católica de Chile, Santiago, Chile.

Roach, A. J., Frazier, L. P. \& Bowden, S. R. (1981). The Marital Satisfaction Scale: Development of a measure for intervention research. Journal of Marriage and Family, 43, 537-546. doi:10.2307/351755

Shaver, P. R., Collins, N. \& Clark, C. L. (1996). Attachment styles and internal working models of self and relationship partners. En G. J. O. Fletcher \& J. Fitness (Eds.), Knowledge structures in close relationships: A social psychological approach (pp. 25-61). Mahwah, NJ: Lawrence Erlbaum.

Simpson, J. A. (1990). Influence of attachment styles on romantic relationships. Journal of Personality and Social Psychology, 59, 971-980. doi:10.1037/0022-3514.59.5.971

Tapia, L. (2001). Algunas consideraciones para una terapia de pareja basada en la evidencia. De Familias y Terapias, 14 y $15,7-30$.

Tapia, L. (2005). La investigación en terapia familiar o de pareja (TFP) o haga usted mismo su propia investigación. De Familias y Terapias, 20, 59-88.

Vera, J., Félix-Castro, J. \& Rodríguez-Barreras, A. (2001). Satisfacción marital, edad, número de hijos y años en pareja. Revista de Psicología, Universidad Mayor de San Marcos, 1-2, 233-250.

Vetere, A. \& Dallos, R. (2008). Systemic therapy and attachment narratives. Journal of Family Therapy, 30, 374-385. doi:10.1111/j.1467-6427.2010.00497_1.x

Wei, M., Vogel, D. L., Ku, T. -Y. \& Zakalik, R. A. (2005). Adult attachment, affect regulation, negative mood, and interpersonal problems: The mediating roles of emotional reactivity and emotional cutoff. Journal of Counseling Psychology, 52, 14-24. doi:10.1037/0022-0167.52.1.14

Fecha de recepción: Agosto de 2011.

Fecha de aceptación: Enero de 2012. 